\title{
Randomised trial of HPV self-sampling among non-attenders in the Slovenian cervical screening programme ZORA: comparing three different screening approaches
}

\author{
Urska Ivanus ${ }^{1,2}$, Tine Jerman ${ }^{1}$, Alenka Repse Fokter ${ }^{3}$, Iztok Takac ${ }^{4}$, \\ Veronika Kloboves Prevodnik ${ }^{5}$, Mateja Marcec ${ }^{4}$, Ursula Salobir Gajsek ${ }^{6}$, Maja Pakiz ${ }^{4}$, \\ Jakob Koren ${ }^{6}$, Simona Hutter Celik ${ }^{7}$, Kristina Gornik Kramberger ${ }^{7}$, Ulrika Klopcic ${ }^{5}$, \\ Rajko Kavalar ${ }^{7}$, Simona Sramek Zatler ${ }^{3}$, Biljana Grcar Kuzmanov8, Mojca Florjancic ${ }^{1}$, \\ Natasa Nolde ${ }^{5}$, Srdjan Novakovic ${ }^{9}$, Mario Poljak ${ }^{10}$, Maja Primic Zakelj ${ }^{1}$

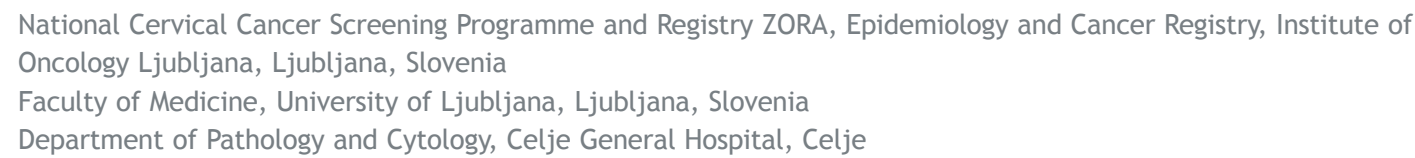

Correspondence to: Urška Ivanuš M.D., Ph.D., National Cervical Cancer Screening Programme and Registry ZORA, Epidemiology and Cancer Registry, Institute of Oncology Ljubljana, Zaloška 2, SI-1000 Ljubljana, Slovenia. Phone: +386 41 901 478; E-mail: uivanus@onko-i.si

Disclosure: No potential conflicts of interest were disclosed.

Background. To overcome obstacles within the Slovenian organised cervical cancer screening programme, a randomised pilot study of human papillomavirus (HPV) self-sampling among non-attenders was performed, aiming to assess three different screening approaches.

Participants and methods. Non-attenders aged 30-64 years from two Slovenian regions were randomised to two HPV self-sampling groups-the opt-in $(I 1, n=14.400)$ and the opt-out $(12, n=9.556)$, with a control group $(P, n=2.600)$. Self-collected samples were analysed using the Hybrid Capture 2 assay. HPV-positive women were invited to a colposcopy. The overall and type-specific intention-to-screen response rates and histological outcomes with a positive predictive value (PPV) according to the women's age, the screening approach, the level of protection resulting from previous screening history, and the region of residence were assessed.

Results. Of the 26.556 women enrolled, 8.972 (33.8\%) responded with self-sample for HPV testing and/or traditional cytology within one year of enrolment. Response rates were $37.7 \%, 34.0 \%$ and $18.4 \%(p<0.050)$ for opt-out, opt-in and control groups. Cervical intraepithelial neoplasia (CIN)2+ was diagnosed in 3.9/1.000, 3.4/1.000, and 3.1/1.000 women ( $p>0.050$ ), respectively. PPV of the HPV self-sampling was $12.0 \%$ and $9.6 \%$ for CIN2+ and CIN3+. The highest PPV was obtained in non-attenders in screening programme for more than 10-years and concordant results of HPV testing with $40.8 \%$ for $\mathrm{CIN} 2+$ and $38.8 \%$ for $\mathrm{CIN} 3+$.

Conclusions. The results of our study show that a high response to HPV self-sampling can be achieved also in an opt-in approach, if women are encouraged to choose between self-sampling at home and screening with gynaecologist. In addition, clinically important risk difference for a high-grade cervical lesion exists in the case of a positive result of HPV testing on self-collected samples, depending on the length of the interval since last screening. Stratified management of these women should be strongly considered. Women who were not screened with cytology for at least 10 years should be referred to immediate colposcopy for histology verification instead to delayed re-testing.

Key words: cervical cancer; screening programme; non-attenders; cytology; HPV; self-sampling 


\section{Introduction}

Cervical cancer screening programmes have successfully reduced the incidence of and mortality from cervical cancer in several countries. ${ }^{1-3}$ In well-organised population-based programmes with good screening test coverage, around half or more of new cases are detected in non-attenders, who usually represent the minority of the eligible population. ${ }^{4}$ Furthermore, these cancers are more often diagnosed in advanced stages, requiring more invasive treatment and subsequently leading to lower quality of life and survival..$^{5-7}$ Similar findings were identified in Slovenia after the implementation of the national, population-based, organised cervical cancer screening national programme (NP) ZORA. ${ }^{8}$

The NP ZORA was implemented in 2003 with conventional cytology in three-year screening intervals for women aged 20-64 years. The three-year coverage of the target population with a screening test is just above $70 \%$, and its five-year coverage is just above $80 \%{ }^{8}$ Higher coverage is observed in younger women and reaches $80 \%$ in the $20-24$ age group. It drops below $70 \%$ in women above 50 and is just below $55 \%$ in the oldest age group of women aged from 60 to 64 . Coverage also varies between administrative units. ${ }^{8}$ Slovenia is among the European countries with the highest historical cervical cancer incidence rates but is also among the countries with the most pronounced decline in cervical cancer incidence rates over time. ${ }^{9}$ The steepest decline was observed after the implementation of the NP ZORA, with the annual change being $-5.8 \%$ in $2003-2015 .{ }^{3}$ Non-attenders in the NP ZORA account for $50-60 \%$ of new cervical cancer cases, among which more than $80 \%$ are diagnosed at FIGO stage II or higher, compared to regular attenders where only $20 \%$ of cancers are diagnosed at stage II or higher. ${ }^{10}$

Non-attendance is the main limiting factor for achieving the full health benefit of screening programmes at the population level in a well-organised, high-quality screening setting. Recent systematic reviews and meta-analyses have shown that offering human papillomavirus (HPV) self-sampling at home to non-attenders can significantly increase attendance and the detection of high-grade cervical lesions, compared to currently widely used reminder letters for clinician-based screening. ${ }^{11}$ Consequently, the recently renewed European guidelines for quality assurance in cervical cancer screening ${ }^{12}$ recommend that countries with organised screening assess whether HPV self-sampling is feasible and cost-effective for non-attenders in the local setting. If it is, countries are encouraged to upgrade their existing screening programmes, but only if they can provide careful monitoring and evaluation of the desired performance and outcomes after implementation. Since HPV selfsampling has similar sensitivity as cytology but lower sensitivity than HPV testing of practitionerobtained cervical samples ${ }^{13}$, HPV self-sampling is recommended only for non-attenders in the organised programmes and not for all women. ${ }^{12}$

There are two main approaches to self-sampling, and there is lack of evidence which of them has a higher overall effect among non-attenders and is more cost-effective. With the opt-out approach, self-sampling devices (testers) are sent by regular mail to non-attenders, while with the optin approach, women are invited to order a tester or a tester can be picked up at a local pharmacy. ${ }^{11}$ Since response rates in early randomised opt-in studies were comparable to control groups and much lower than in opt-out studies ${ }^{14-16}$, the opt-in approach was not favoured in further studies. ${ }^{11}$ When implementing a new intervention at the population level, however, it is necessary to keep costs as low as possible. Due to high wastage of distributed testers, the opt-out approach might be less feasible in terms of cost-effectiveness, while the opt-in approach mitigates this problem. To the best of our knowledge, only two studies have been published until now that suggest that the opt-in approach could generate a high response. A recent Swedish study showed a significantly higher response in opt-in self-sampling compared to both a reminder letter and a reminder telephone call, but the study did not include an opt-out group. ${ }^{16}$ Only one, very recent, randomised study, conducted in Denmark, showed a higher response in an opt-in self-sampling group compared to a control group, but even there the response was lower than in the opt-out group. ${ }^{17}$

The available evidence supports the implementation of HPV self-sampling in countries with wellorganised screening, such as Slovenia. The aim of the present study, performed among non-attenders in the NP ZORA, was to compare three approaches (opt-out, opt-in and a reminder letter for screening at the clinic) in a randomised setting within an ongoing, population-based screening programme with relatively high coverage. Two main research questions were probed: (1) is it possible to reach an adequate response rate in the opt-in compared opt-out approach if women are given the option to order a tester for HPV self-sampling or visit a 
gynaecologist for cytology and are encouraged to select their preferred option? and (2) should the same follow-up diagnostic procedure be used for all women following a positive result of HPV test performed on self-collected samples taken at home or should a stratified approach be considered?

\section{Participants and methods}

\section{Study protocol}

The study was designed as a pilot for HPV selfsampling among non-attenders in the NP ZORA. It was an open label, multi-arm study with a randomised design. Study protocol is summarised below; full protocol is described in details in Supplementary material (SupMat 1 ).

Women were eligible for the study if they were aged 30-64, had no cytology result registered in the National Cervical Cancer Screening Registry (the ZORA registry) in the last four years, had their permanent residence in the Celje or Maribor regions, and had no recorded hysterectomy in the ZORA registry. Total of 26.556 eligible women were randomly selected from the ZORA registry and randomly allocated to the opt-in (I1, n = 14.400), optout $(\mathrm{I} 2, \mathrm{n}=9.956)$ or control $(\mathrm{P}, \mathrm{n}=2.600)$ groups (Figure 1). The eligible women were randomly selected and allocated once per month, using a random number generator in R. ${ }^{18}$ The randomisation was stratified by five-year age groups, screening history and region of residence. Ten random selections and allocations were done in 2015, as described in the supplementary material (SupMat 1). Invitation letters with attached questioner for double-checking the eligibility criteria were sent within a month of the enrolment to all selected women, between 6 January and 1 December 2015. Women were excluded from further correspondence in three distinct stages; after the initial letter was sent but before a tester was sent, after a tester was sent or after the result of the HPV test performed on a self-collected sample was sent (Figure 1).

During the study, all the women had free access to regular cytological screening with their personal gynaecologist (PG), who are the providers of cervical screening in Slovenia. Women in the intervention groups were invited to take a cervicovaginal sample with a self-sampling device (tester) at their home, following the manufacturer's instructions, and send it to the laboratory by ordinary mail as soon as possible.

Three different testers were used in the optout study group: Qvintip (Aprovix AB, Uppsala,
Sweden), HerSwab (Eve Medical, Toronto, Canada) and Delphi Screener (Delphi Bioscience, Scherpenzeel, the Netherlands). In the opt-in study group, only Qvintip was used. All self-collected samples were tested for the presence of 13 highrisk HPV types using the clinically validated test Hybrid Capture 2 High-Risk HPV DNA assay (HC2, Qiagen, Hilden, Germany), following the manufacturer's instructions. The cut-off value of $\mathrm{RLU} / \mathrm{CO}=1.00 \mathrm{pg} / \mathrm{ml}$ was used to distinguish between positive and negative HPV results. Selfcollected samples were evaluated for cellularity control by visual pellet assessment after centrifugation. Self-collected samples with negative HC2 result and no visual pellet were processed further. The DNA concentration was measured and, if it was below a cut-off point of $5 \mathrm{ng} / \mathrm{ul}$, the sample was regarded as technically inadequate. Cytological and histological evaluations were performed by certified pathologists participating in the NP ZORA.

The result of the HPV test and further recommendations were sent to all responders. If the result was HPV negative, it was recommended to the woman that she attend a regular cytological screening examination after three years. All women with a HPV positive result were offered an appointment for a colposcopy at a regional hospital (University Clinical Centre Maribor or Celje General Hospital). Women with technically inadequate self-collected samples received invitation to visit their PG. In the case of an abnormal colposcopy result, a biopsy was taken; no blind biopsies were taken. Women were managed according to the national guidelines. ${ }^{19}$

The study was coordinated by the Institute of Oncology Ljubljana. It was conducted in compliance with the Helsinki Declaration and was approved by the National Medical Ethics Committee at the Slovenian Ministry of Health (consents Nos. $155 / 03 / 13$ and 136/04/14). It was financed by the Slovenian Research Agency and the Slovenian Ministry of Health (trial No. L3-5512).

\section{Primary and secondary outcomes}

The primary outcomes were: (1) intention-to-screen response rates among all enrolled women and (2) high-grade histology outcomes among all enrolled women and all responders according to the age of the woman, opt-in and the opt-out approach, region of residence and the level of protection resulting from previous screening history. The intentionto-screen response rate was computed as a proportion of enrolled women (all women who were 
FIGURE 1. Flow diagram of 26.556 non-attenders in the organised Slovenian cervical cancer screening programme ZORA, randomly selected from a screening registry for pilot implementation of HPV self-sampling and randomly allocated to the opt-in (I1), opt-out (I2) and control (P) study group.
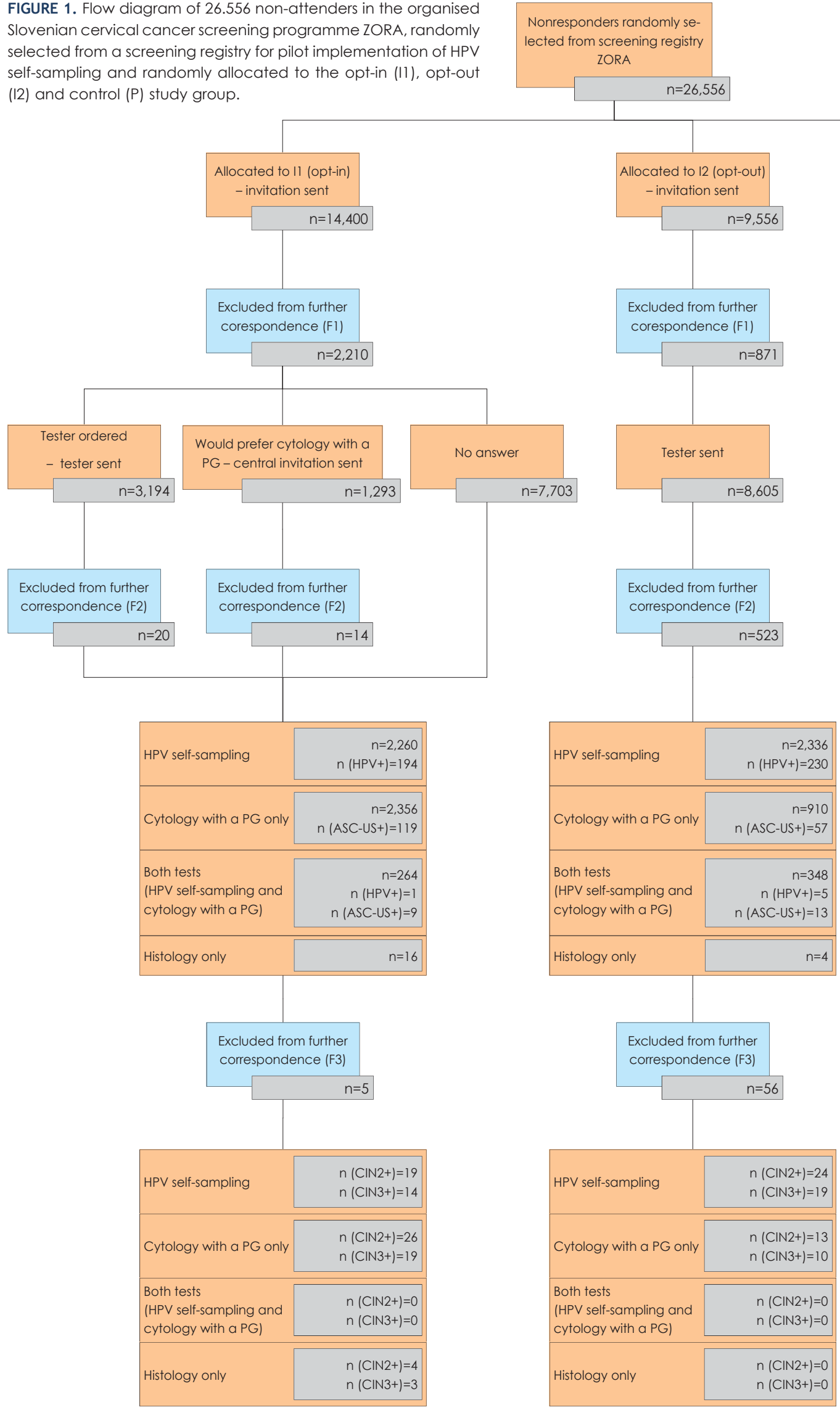

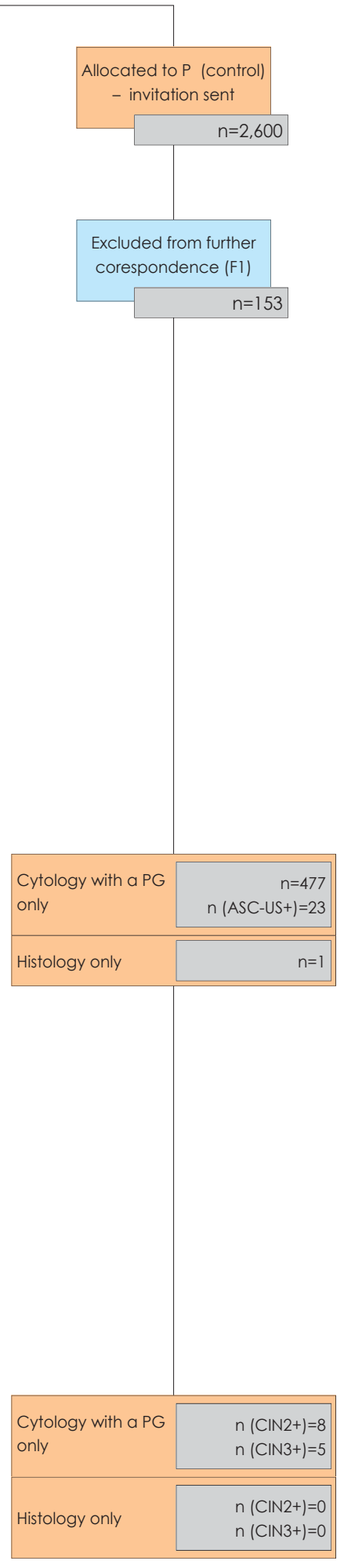


randomly selected from the ZORA registry) with a response within one year after the enrolment. The response was defined as having an HPV test performed on self-collected sample and/or cytology with PG within one year after the enrolment. The response types were classified as 'HPV self-sampling only' (type A response), 'cytology screening with a PG only' (type B response), or 'both tests' (both HPV test on self-collected sample and cytology screening with a PG, type $C$ response). Very few women visited PG for a cytological screening after the enrolment, but only a histological sample was taken from the cervix, mostly because the changes of the cervix were already visible. These women were considered type B responders. The high-grade histological outcome was defined as the diagnosis of a cervical intraepithelial neoplasia (CIN)2+ or CIN3+ lesion within one year after enrolment in the study. High-grade histology outcomes were computed as a proportion of women with the CIN2+ or CIN3+ outcome among all the enrolled women and among the responders. PPV of the HPV test was computed as a proportion of women with a high-grade histological outcome among all the women who attended colposcopy after a positive HPV test. Cytological and histological outcomes within one year after the enrolment were identified for all the enrolled women by linking the study database with the ZORA registry. If a woman had more than one histological outcome, the lesion with the highest grade was used in calculations.

Secondary outcomes were: (1) tester ordering in the opt-in study group, (2) the results of the HPV test on self-collected sample, (3) the compliance at further examinations for women with a positive HPV test, and a (4) positive concordance of the result of the HPV testing on self-collected samples and on samples taken by a practitioner. The tester ordering was computed as a proportion of the women enrolled in the opt-in study group who opted in for a tester among all the women enrolled in opt-in study group. The results of screening tests are presented as the proportions of positive and the proportions of technically inadequate self-collected samples. The compliance was computed as the proportion of all the women who attended the examination either at a regional clinic or with their PG from all the women with a positive HPV test performed on self-collected sample. The results of the HPV testing on self-collected samples and on practitioner-obtained samples were concordant if both were positive at the cut-off value RLU/CO = $1.00 \mathrm{pg} / \mathrm{ml}$.

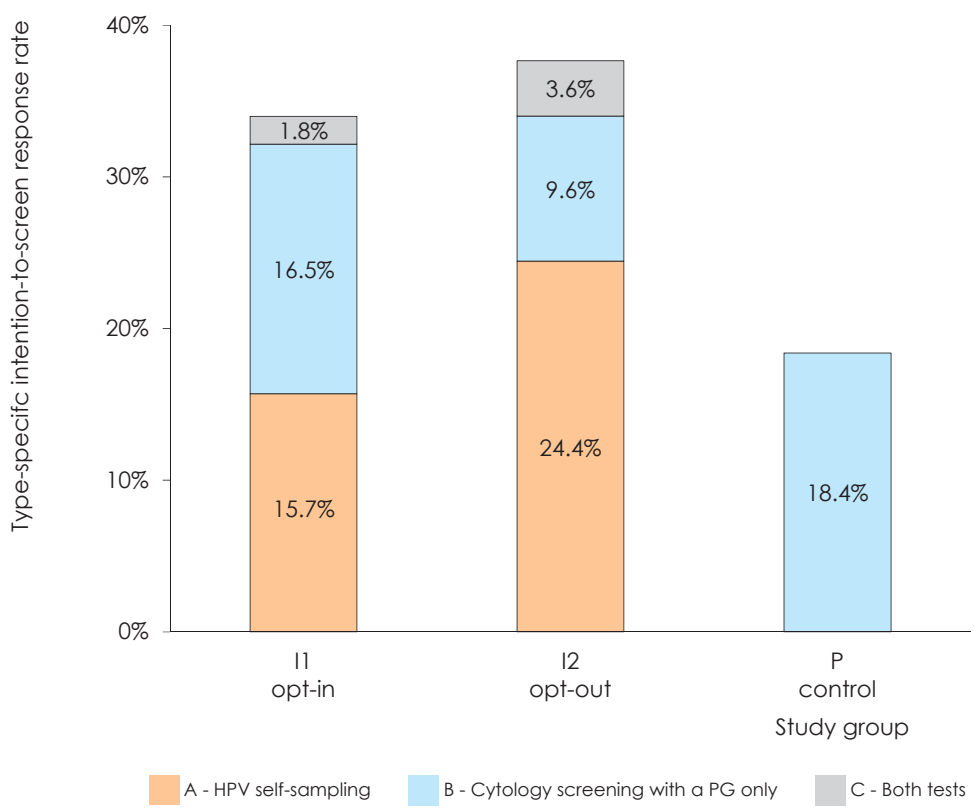

FIGURE 2. Intention-to-screen response rate in study groups opt-in (11), opt-out (I2) and comparison (P) by three types of response: HPV self-sampling (type A response), cytology screening with a PG only (type B response) or both (HPV self-sampling and cytology screening with a PG only, type $C$ response).

\section{Statistical analyses}

Response rates were analysed only on the intention-to-screen basis. The effect size was estimated as a relative risk ratio within the $95 \%$ confidence interval (CI). Predefined subgroup analyses of possible significant predictors for the response and histological outcomes were performed with the aim to identify common characteristics of responders and approaches with the highest response rate and high-grade lesions detection (Table 2 and Table 3). The univariate logistic regression was used to assess if the outcome was significantly associated with the possible predictor. The multivariate logistic regression analysis was performed for all primary and secondary outcomes to adjust the outcome for predefined predictors. The predefined predictors included in these analyses were the woman's age, the region of residence, the level of protection, and the study group. The data on predictors were obtained from the ZORA registry at the time of the random selection. The level of protection due to previous screening history was categorised as 'medium protection' if the last cytology was done 4-9 years prior to the enrolment and as 'no/low protection' if the last cytology was done 10 years or more prior to the enrolment or if the woman didn't have any cytology result in the ZORA registry. Age was used as a continuous variable in all univariate and 
multivariate analyses. In the subgroup analysis, women's age was described in terms of the mean and the $95 \%$ confidence intervals. Other possible predictors were described as proportions; chisquare test was used to determine if the observed difference in the distribution of proportions within subgroups was statistically significant. Additional analyses were performed to explore type-specific response rates (Figure 2), high-grade disease outcomes among responders, and high-grade disease outcomes in subgroups of women with the highest PPV for high-grade disease. All analyses were conducted with SPPS 22.0 (SPSS; Chicago, IL, USA) and the open source programme language $R$, using 2-tailed tests and the significance level $\alpha=0.050 .{ }^{18}$ We have used the random number generator in the programme language $\mathrm{R}$ for the study group selection, programme language $\mathrm{R}$ was also used for the calculations of relative risk ratios and their 95\% confidence intervals.

\section{Results}

\section{Characteristics of women}

In total, 26.556 non-attenders in the Slovenian organised cervical cancer screening programme ZORA were randomly selected and randomly allocated to three study groups: the opt-in group (I1, $\mathrm{n}$ $=14.400)$, the opt-out group $(\mathrm{I} 2, \mathrm{n}=9.556)$, and the control group $(\mathrm{P}, \mathrm{n}=2.600)$ (Figure 1).

Table 1 shows the characteristics of the enrolled women. The mean age was 49.8 years (95\% CI: 49.7-50.0) and was similar across all study groups. $41.6 \%$ of women were permanently residing in the Celje region and $58.4 \%$ in the Maribor region. More than half of the enrolled women $(53.1 \%)$ had no/ low level of protection. The distribution of women

TABLE 1. Characteristics of women enrolled in the study

\begin{tabular}{|c|c|c|c|c|c|c|}
\hline \multirow{2}{*}{$\begin{array}{c}\text { All randomly } \\
\text { selected and } \\
\text { allocated women } \\
\end{array}$} & \multicolumn{2}{|c|}{ Total } & \multicolumn{4}{|c|}{ STUDY GROUPS } \\
\hline & Number & $\%$ & $\begin{array}{c}11 \\
\text { opt-in }\end{array}$ & $\begin{array}{c}12 \\
\text { opt-out }\end{array}$ & $\begin{array}{c}\mathrm{P} \\
\text { control } \\
\end{array}$ & P-value \\
\hline Number & 26,556 & 100.0 & 14,400 & 9,556 & 2,600 & \\
\hline $\begin{array}{l}\text { Age } \\
\text { Mean age }(95 \% \mathrm{Cl})\end{array}$ & $\begin{array}{c}49.8 \\
(49.7-50.0)\end{array}$ & & $\begin{array}{c}49.8 \\
(49.7-50.0)\end{array}$ & $\begin{array}{c}49.8 \\
(49.6-50.0)\end{array}$ & $\begin{array}{c}50.0 \\
(49.6-50.3)\end{array}$ & \\
\hline \multicolumn{7}{|l|}{ Level of protection } \\
\hline medium & 12,464 & 46.9 & 6,796 & 4,540 & 1,128 & \multirow{2}{*}{$0.001^{*}$} \\
\hline no/low & 14,092 & 53.1 & 7,604 & 5,016 & 1,472 & \\
\hline \multicolumn{7}{|l|}{ Region } \\
\hline Celje & 11,055 & 41.6 & 5,996 & 3,984 & 1,075 & \multirow{2}{*}{0.951} \\
\hline Maribor & 15,501 & 58.4 & 8,404 & 5,572 & 1,525 & \\
\hline
\end{tabular}

*Statistically significant result at $a=0.05$. according to the level of protection differed significantly between the study groups, most likely due to the refreshment of the target population with new women with medium protection who became eligible for the study between samplings and because different study groups were included at different samplings (SupMat 1).

Altogether 3.852 (14.5\%) women were excluded from further correspondence at different stages during the study after the initial invitation letter with a questionnaire was sent (Figure 1). Major reasons were a previous unregistered hysterectomy reported by woman via questionnaire attached to the study invitation letters $(4.0 \%, 1.075 / 26.556)$, undelivered invitation letter due to unknown address or unaccepted mail $(3.6 \%, 947 / 26.556)$, recent cytology not yet registered in the ZORA registry $(2.2 \%$, $591 / 26.556)$, living abroad $(2.0 \%, 537 / 26.556)$, rejection of participation in the study $(1.9 \%, 500 / 26.556)$, or other reasons, such as virginity, severe disability, current pregnancy, or other $(0.8 \%, 202 / 26.556)$.

\section{Response}

Out of 26.556 enrolled women, 8.972 (33.8\%) selfcollected a sample for the HPV test or attended screening with their PG up to one year after the initial invitation letter was sent. In the univariate analysis, the overall response rate was significantly associated with the study groups, age of women and the level of protection, but not to the region of residence. After the adjustment in the multivariate analysis, the overall response was significantly associated with all the predictors.

Table 2 shows intention-to-screen response rates in study groups. The response rates were significantly different among all study groups. Response was 2.0-times higher (95\% CI: 1.9-2.2) in the optout study group than in the control study group (37.7\% vs. $18.4 \%)$, 1.8 -times higher (95\% CI: $1.7-2.0$ ) in the opt-in study group than in the control study group (34.0\% vs. $18.4 \%$ ) and 1.11-times higher $(95 \%$ CI: 1.07-1.15) in the opt-out study group than in the opt-in study group (37.7\% vs. 34.0\%). Women with medium protection had a 2.8 -times higher $(95 \%$ CI: 2.7-2.9) response rate than women with no/low protection (51.1\% vs. $18.5 \%)$. The responders from the Maribor region were also statistically significantly older than responders from the Celje region.

Response rates also remained significantly different among study groups in a stratified analysis of the subgroup of women with medium and with no/low protection due to the past screening history (Table 3). In the subgroup of women with no/ 
TABLE 2. The main results by their predictors. Intention-to-screen response rates, the mean age of responders and histological outcomes are presented as absolute numbers, proportions (per 100 or 1.000) and $95 \%$ confidence intervals (CI) by the study group, the region of residence and the level of protection

\begin{tabular}{|c|c|c|c|c|c|c|c|c|c|}
\hline & & & & Intention to & & & ion to screen & olog & outcome \\
\hline & & & of & response rate & $(95 \% \mathrm{Cl})$ of & & L/CIN2+ & & L/CIN3+ \\
\hline & & & women & $\begin{array}{l}\text { per } 100(\%) \\
\text { with } 95 \% \mathrm{Cl}\end{array}$ & & no. & $\begin{array}{l}\text { per } 1000(\% \circ) \\
\text { with } 95 \% \mathrm{Cl}\end{array}$ & no. & $\begin{array}{c}\text { per } 1000(\% \circ) \\
\text { with } 95 \% \mathrm{Cl}\end{array}$ \\
\hline ঊ & $\begin{array}{l}\text { All w } \\
\text { Resp }\end{array}$ & omen & $\begin{array}{l}26,556 \\
8,972\end{array}$ & $\begin{array}{c}33.8 \% \\
(33.2 \%-34.4 \%)\end{array}$ & $\begin{array}{c}49.0 \\
(48.7-49.2)\end{array}$ & 94 & $\begin{array}{c}3.5 \% \circ \\
(2.9 \%-4.4 \% \circ)\end{array}$ & 71 & $\begin{array}{c}2.7 \% \circ \\
(2.1 \% \circ-3.4 \% \circ)\end{array}$ \\
\hline & $\begin{array}{c}11 \\
\text { opt-in }\end{array}$ & $\begin{array}{c}\text { all } \\
\text { responders }\end{array}$ & $\begin{array}{l}14,400 \\
4,896\end{array}$ & $\begin{array}{c}34.0 \% \\
(33.2 \%-34.8 \%)\end{array}$ & $\begin{array}{c}49.0 \\
(48.7-49.3)\end{array}$ & 49 & $\begin{array}{c}3.4 \% \circ \\
(2.5 \% \circ-4.5 \% \circ)\end{array}$ & 36 & $\begin{array}{c}2.5 \% \circ \\
(1.8 \% \circ-3.5 \% \circ)\end{array}$ \\
\hline 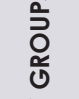 & $\begin{array}{c}12 \\
\text { opt-out }\end{array}$ & $\begin{array}{c}\text { all } \\
\text { responders }\end{array}$ & $\begin{array}{l}9,556 \\
3,598\end{array}$ & $\begin{array}{c}37.7 \% \\
(36.7 \%-38.6 \%)\end{array}$ & $\begin{array}{c}49.0 \\
(48.6-49.3)\end{array}$ & 37 & $\begin{array}{c}3.9 \% \circ \\
(2.8 \% \circ-5.4 \% \circ)\end{array}$ & 30 & $\begin{array}{c}3.1 \% \circ \\
(2.2 \% \circ-4.5 \% \circ)\end{array}$ \\
\hline 点 & $\begin{array}{c}P \\
\text { control }\end{array}$ & $\begin{array}{c}\text { all } \\
\text { responders }\end{array}$ & $\begin{array}{r}2,600 \\
478\end{array}$ & $\begin{array}{c}18.4 \% \\
(16.9 \%-19.9 \%)\end{array}$ & $\begin{array}{c}48.0 \\
(47.2-48.9)\end{array}$ & 8 & $\begin{array}{c}3.1 \% \circ \\
(1.4 \% \circ-6.3 \% \circ)\end{array}$ & 5 & $\begin{array}{c}1.9 \% \circ \\
(0.7 \% \circ-4.8 \% \circ)\end{array}$ \\
\hline & & ylue & & $<0.000^{*}$ & & & 0.766 & & 0.557 \\
\hline & Celje & $\begin{array}{c}\text { all } \\
\text { responders }\end{array}$ & $\begin{array}{l}11,055 \\
3,666\end{array}$ & $\begin{array}{c}33.2 \% \\
(32.3 \%-34.0 \%)\end{array}$ & $\begin{array}{c}48.3 \\
(47.9-48.6)\end{array}$ & 38 & $\begin{array}{c}3.4 \% \circ \\
(2.5 \%-4.8 \% \circ)\end{array}$ & 28 & $\begin{array}{c}2.5 \% \circ \\
(1.7 \% \circ-3.7 \% \circ)\end{array}$ \\
\hline 矛 & Maribor & $\begin{array}{c}\text { all } \\
\text { responders }\end{array}$ & $\begin{array}{r}15,501 \\
5,306\end{array}$ & $\begin{array}{c}34.2 \% \\
(33.5 \%-35.0 \%)\end{array}$ & $\begin{array}{c}49.4 \\
(49.2-49.7)\end{array}$ & 56 & $\begin{array}{c}3.6 \% \\
(2.8 \% \circ-4.7 \%)\end{array}$ & 43 & $\begin{array}{c}2.8 \% \circ \\
(2.0 \% \circ-3.8 \% \circ)\end{array}$ \\
\hline & & & & 0.070 & & & 0.813 & & 0.708 \\
\hline 으 & Medium & $\begin{array}{c}\text { all } \\
\text { responders }\end{array}$ & $\begin{array}{r}12,464 \\
6,367\end{array}$ & $\begin{array}{c}51.1 \% \\
(50.2 \%-52.0 \%)\end{array}$ & $\begin{array}{c}48.4 \\
(48.2-48.7)\end{array}$ & 48 & $\begin{array}{c}3.9 \% \circ \\
(2.9 \% \circ-5.1 \% \circ)\end{array}$ & 33 & $\begin{array}{c}2.6 \% \circ \\
(1.9 \% \circ-3.8 \% \circ)\end{array}$ \\
\hline 苋 & No/low & $\begin{array}{c}\text { all } \\
\text { responders }\end{array}$ & $\begin{array}{l}14,092 \\
2,605\end{array}$ & $\begin{array}{c}18.5 \% \\
(17.8 \%-19.1 \%)\end{array}$ & $\begin{array}{c}51.1 \\
(50.9-51.3)\end{array}$ & 46 & $\begin{array}{c}3.3 \% \circ \\
(2.4 \% \circ-4.4 \% \circ)\end{array}$ & 38 & $\begin{array}{c}2.7 \% \circ \\
(1.9 \% \circ-3.7 \% \circ)\end{array}$ \\
\hline 凹 & & & & $<0.000 *$ & & & 0.422 & & 0.838 \\
\hline
\end{tabular}

*Statistically significant result at $a=0.05$.

low protection, the response was also significantly higher in the Maribor (19.5\%) than the Celje region $(17.0 \%)$.

\section{Type-specific response}

Out of 26.556 enrolled women, 4.596 (17.3\%) women performed only HPV self-sampling, (type A response), $3.764(14.2 \%)$ attended screening with their PG only (type B response) and 612 (2.3\%) women performed both HPV self-sampling and attended the screening with the selected PG (type C response). In total, 5.208 HPV tests performed on self-collected samples $(4.596+612)$ and 4.355 cytological results $(3.743+612)$ were registered during the study. Among 3.764 women with the type B response, 3.743 had the cytology result recorded and 21 had only the histology result recorded (without cytology). Most women with the type $C$ response first had the HPV testing on self-collected sample and later decided to additionally attend the cytological screening with their PG $(92.6 \%, 567 / 612)$.

Figure 2 shows the intention-to-screen response rate by the type of response in all the three study groups. Distribution of type A and type B response rates in opt-in and opt-out group differed in such 
way, that type A response was statistically significantly higher in opt-out compared to opt-in group ( $24.4 \%$ vs. $15.7 \%$, 95\% CI do not overlap) and contrary, the type B response was statistically significantly higher in opt-in compared to opt-out group (16.5\% vs. 9.6\%, 95\% CI do not overlap) and at the same time comparable to the response in the control group (18.4\%, overlapping 95\% CI with the opt-in but not with the opt-out study group) (Table 2 at SupMat2). Only a small proportion of women $-1.8 \%$ in the opt-in study group and $3.6 \%$ in the opt-out study group - had the type C response. The supplementary material provides further data regarding the statistically significant differences in the type $\mathrm{A}, \mathrm{B}$ and $\mathrm{C}$ response rates among the study groups (SupMat 2).

\section{Tester order in the opt-in group}

Out of 14.400 women in the opt-in study group, $22.2 \%(3.194 / 14.400 ; 95 \%$ CI: $21.5 \%-22.9 \%)$ ordered the tester. The ordering was significantly associated with the region and the level of protection. It was 1.1-times higher (95\% CI: 1.1-1.2) in the Maribor than the Celje region $(23.4 \%$ vs. $20.4 \%$ ), and 1.6-times higher (95\% CI: $1.5-1.7)$ in women with medium compared to no/low protection $(27.4 \%$ vs. $17.5 \%)$. Out of 3.194 women who ordered the tester, 2.524 (79.0\%; 95\% CI: $77.6 \%-$ $80.4 \%)$ returned the self-collected sample. The

TABLE 3. Response rate stratified by the level of protection due to previous screening. Intention-to-screen response rates are presented as absolute numbers, proportions (per 100) and $95 \%$ confidence intervals (CI) by the study group and the region of residence
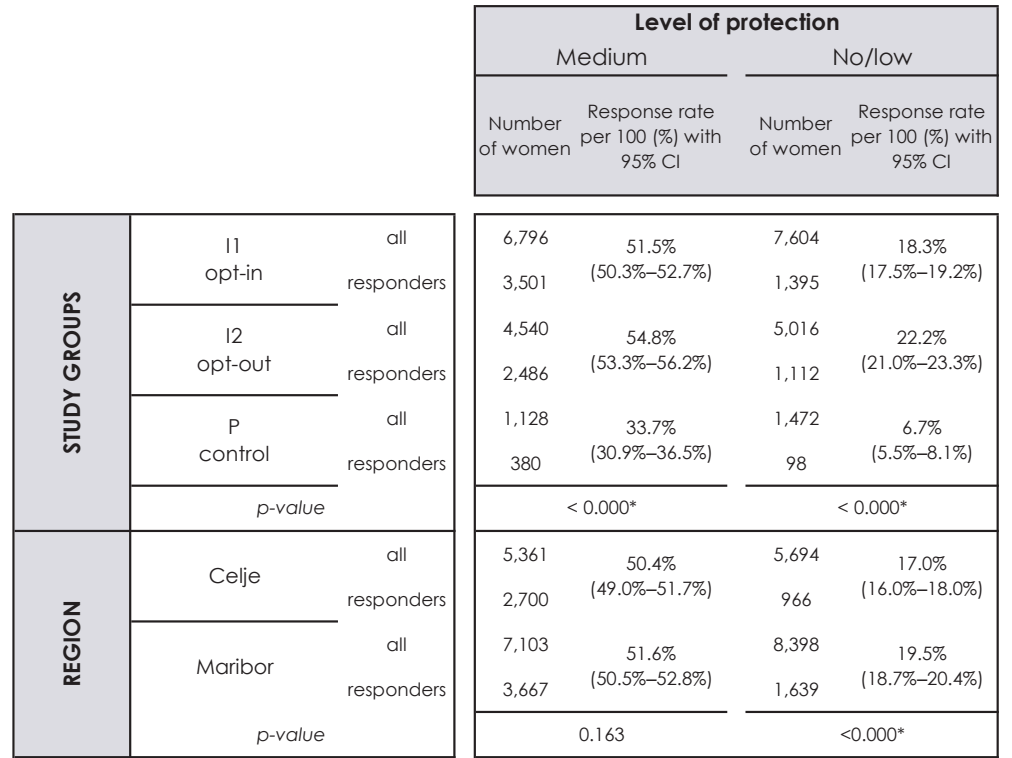

\begin{tabular}{|c|c|c|c|}
\hline 6,796 & \multirow{2}{*}{$\begin{array}{c}51.5 \% \\
(50.3 \%-52.7 \%)\end{array}$} & 7,604 & \multirow{2}{*}{$\begin{array}{c}18.3 \% \\
(17.5 \%-19.2 \%\end{array}$} \\
\hline 3,501 & & 1,395 & \\
\hline 4,540 & \multirow{2}{*}{$\begin{array}{c}54.8 \% \\
(53.3 \%-56.2 \%)\end{array}$} & 5,016 & \multirow{2}{*}{$\begin{array}{c}22.2 \% \\
(21.0 \%-23.3 \%)\end{array}$} \\
\hline 2,486 & & 1,112 & \\
\hline 1,128 & $\begin{array}{c}33.7 \% \\
(30.9 \%-36.5 \%)\end{array}$ & & $\begin{array}{c}6.7 \% \\
(5.5 \%-8.1 \%)\end{array}$ \\
\hline \multicolumn{2}{|c|}{$<0.000^{*}$} & \multicolumn{2}{|c|}{$<0.000^{*}$} \\
\hline 5,361 & $\begin{array}{c}50.4 \% \\
(49.0 \%-51.7 \%)\end{array}$ & 5,694 & $\begin{array}{c}17.0 \% \\
(16.0 \%-18.0 \%)\end{array}$ \\
\hline 7,103 & $\begin{array}{c}51.6 \% \\
(50.5 \%-52.8 \%)\end{array}$ & 1,639 & $\begin{array}{c}19.5 \% \\
(18.7 \%-20.4 \%)\end{array}$ \\
\hline & 0.163 & & $<0.000^{*}$ \\
\hline
\end{tabular}

*Statistically significant result at $a=0.05$ response after the test order was significantly associated with the age and the level of protection. It was higher in elderly women and women with medium protection $(81.6 \%, 1.520 / 1.863)$ than in younger women and women with low/no protection $(75.4 \%, 1.004 / 1.331)$.

\section{Results of HPV testing on self-collected samples}

The overall HPV positivity rate in the total study population was $8.3 \%$ (95\% CI: $7.5 \%-9.0 \%$ ); the HPV was detected in 430 out of 5.208 self-collected samples (Figure 1). HPV positivity was $7.7 \%$ (195/2.524; 95\% CI: 6.7\%-8.9\%) in the opt-in study group and $8.8 \%(235 / 2.684 ; 95 \%$ CI: $7.7 \%-9.9 \%)$ in the opt-out study group, the difference was not statistically significant. HPV positivity was also not significantly associated with the region and the level of protection. To the contrary, younger women had significantly higher positivity rates than older women in both intervention study groups.

The rate of technically inadequate self-collected samples was $1.4 \%$ (75/5.208; 95\% CI: $1.1 \%-1.8 \%)$. Women with technically inadequate self-collected samples (mean age 56.0; 95\% CI: 54.2-57.8) were significantly older than all the women who selfcollected a sample for the HPV test (mean age 50.3 years; $95 \%$ CI: 50.1-50.6). The HPV test rate of technically inadequate self-collected samples was not associated with the study group, region, or the level of protection.

\section{Compliance with a follow-up examination after a positive HPV test}

Out of 430 women with a positive result of HPV testing on self-collected samples, 388 (90.2\%) complied with a further follow-up examination; 333 $(77.4 \%)$ attended the prescheduled examination at a colposcopy clinic, and $55(12.8 \%)$ visited a PG. 42 $(9.8 \%)$ of women with a positive result of HPV testing on self-collected didn't respond to the invitation for a further follow-up examination. The compliance was significantly higher in women from the Maribor region $(92.9 \%, 234 / 252)$ and women with the medium protection $(94.3 \%, 263 / 279)$ than in women from the Celje region $(86.5 \%, 154 / 178)$ and women with no/low protection $(82.8 \%, 125 / 151)$. The multivariate analysis confirmed that compliance was not associated with the age of the women or the study group.

Out of 333 women who attended a further examination, $146(43.8 \%)$ had a positive result of the 
TABLE 4. Positive predictive value (PPV) of HPV test for $\mathrm{CIN} 2+$ and $\mathrm{CIN} 3+$ in women who had undergone colposcopy after a positive HPV test on self-collected sample and in women with concordant results of both HPV tests. Results are stratified by the level of protection due to previous screening

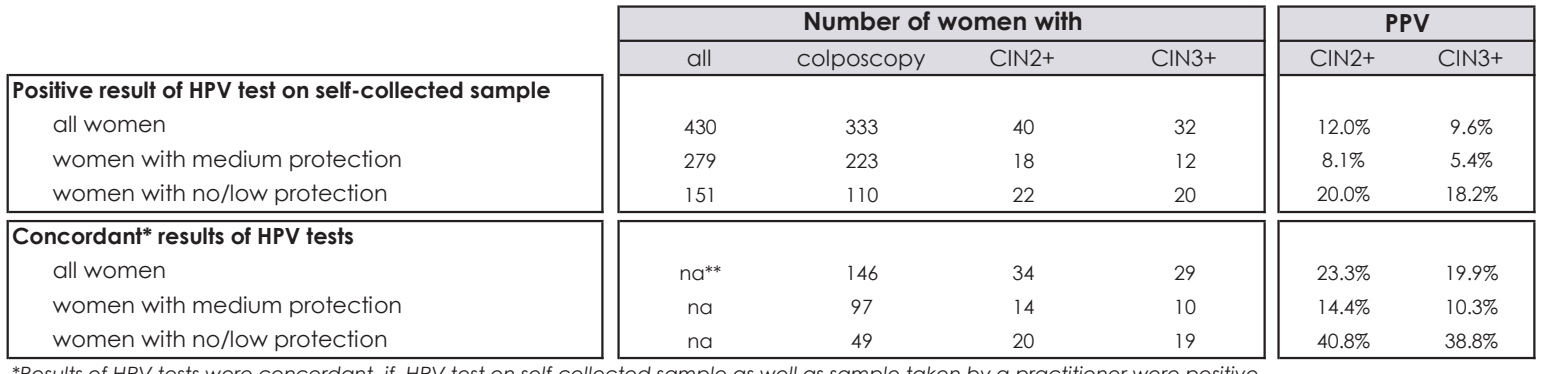

*Results of HPV tests were concordant, if HPV test on self-collected sample as well as sample taken by a practitioner were positive.

**Results not available (na), since only women with colposcopy had a sample taken by a practitioner.

second HPV test carried out on a cervical sample obtained by the practitioner. The follow-up examination took place on mean of 57 days (median 48, range 13-317 days) after the self-collected sample was received in the laboratory by mail. Women with concordant positive results of the HPV testing on self-collected samples and on a practitionerobtained cervical samples were younger (mean age 44.6 years; 95\% CI: 42.9-46.3) than women with discordant results (mean age 49.3 years; $95 \%$ CI: 47.8-50.9). In the multivariate analysis, the concordance was not significantly associated with the study group, region, or the level of protection.

\section{Histological outcomes}

In one year after the enrolment, 94 women were diagnosed with CIN2+ and among those 71 with CIN3+. Among the $94 \mathrm{CIN} 2+$, there were 11 cervical carcinomas ( 3 adenocarcinomas, 8 squamous carcinomas), 59 CIN3, 23 CIN2 and a single case of vaginal intraepithelial neoplasia (VaIN) grade 3. Ten out of eleven cervical carcinomas were diagnosed in women with no/low protection, and one in women with medium protection. Ten out of eleven carcinomas were diagnosed in women enrolled in intervention groups, and one in women enrolled in the control group. Eight of eleven carcinomas were diagnosed in women with the type B response; four of those only had a histological result without cytology. Five carcinomas were diagnosed in FIGO stage I (two in IA1 and three in IA2), four in stage IIB and two in stage IIIB. No CIN2+ was observed in the 612 women who underwent both tests (type $C$ response).

The probability of the CIN2+ or CIN3+ outcome (per 1.000 women, \%o) among 26.556 enrolled women was $3.5 \%$ and $2.7 \%$, respectively (Table 2 ). The probability for the detection of CIN2+ or
CIN3+ after the enrolment was significantly higher in younger women, in both the univariate and multivariate analysis, and it was decreasing with age. The study group, region and level of protection were not significantly associated with the CIN2+ or CIN3+ outcome.

An additional analysis was done to evaluate the detection of CIN2+ and CIN3+ among the responders. The CIN2+ or CIN3+ detection rate in responders was $10.5 \%$ o $(94 / 8.972)$ and $7.9 \%$, respectively (71/8.972) and was significantly associated with age and the level of protection, but not to the study group or region. The detection of CIN2+ or CIN3+ was significantly higher in younger than older responders. The responders with no/low protection had a significantly higher probability for high-grade lesions; $17.7 \%$ o (46/2.605) had CIN2+ and $14.6 \%$ (38/2605) had CIN3+; compared to the responders with medium protection, where $7.5 \%$ o (48/6.367) had CIN2+ and 5.2\%o (33/6.367) CIN3+. The estimated prevalence rate for the invasive cervical cancer was 383.9/100.000 in study responders with no/low protection (10/2.605), compared to $15.7 / 100.000$ in the study responders with medium protection (1/6.367).

\section{Positive predictive value of HPV testing on self-collected samples}

Out of 333 women with a positive result of the HPV test performed on a self-collected sample who had colposcopy at a follow-up examination, 40 (12.0\%) were diagnosed as CIN2+ and $32(9.6 \%)$ as CIN3+ (Table 4). The PPV for CIN2+ and CIN3+ were significantly associated with the level of protection in both univariate and multivariate analyses. The women with no/low protection had 2.5-times higher (20.0\% vs. 8.1\%; 95\% CI: 1.4-4.4) PPV for CIN2+ and 3.4-times higher (18.2\% v 5.4\%; 95\% CI: 1.7-6.7) PPV 
for CIN3+ than women with medium protection. Age was a significant predictor only in the multivariate analysis for CIN2+. The study group and region were not significantly associated with PPV.

PPV was even higher in women with concordant positive HPV test results. Out of 146 women with concordant positive HPV test results, 34 (23.3\%) were diagnosed with CIN2+ and 29 (19.9\%) with CIN3+ (Table 4). Women with no/low protection and concordant positive HPV test results had the highest risk for a high-grade lesion; it was $40.8 \%$ (20/49) for CIN2+ and 38.8\% (19/49) for CIN3+. The risk was significantly higher in this group than in women with medium protection and concordant positive HPV test results, where the corresponding risks were $14.4 \%(14 / 97)$ and $10.3 \%$, respectively (10/97). In the multivariate analysis, this difference also remained significant after adjustment. In women with concordant positive HPV test result, age, study group, and region were not significantly associated with the high-grade histology outcome.

\section{Discussion}

The Slovenian HPV self-sampling study was carried out as a randomised study within an ongoing organised cervical cancer screening programme, with the aim to explore the possible predictors for the intention-to-screen response rates and high-grade histology outcome, including PPV among non-attenders in the programme. Some predictors were related to the intervention (screening approach) and some were related to women's characteristics (age, region of residence, and level of protection from previous screening). In the multivariate analysis, the response rate was significantly associated with all the predictors. It was significantly higher in the opt-out approach compared to the opt-in approach and in the opt-in approach compared to a regular reminder. A significantly better response rate was detected in younger women, women with medium protection and women from the Maribor region. We also observed a difference in the typespecific response between the study groups. The response to attend routine cytology-based screening was higher in the opt-in approach group, while the HPV self-sampling response was higher in the opt-out approach group. In the multivariate analysis, the histology outcome among all the enrolled women was associated only with age, with younger women having a significantly higher probability to be diagnosed with a high-grade lesion. However, the detection of high-grade lesions among respond- ers was significantly associated with age and the level of protection. The responders with no/low protection had significantly higher detection rates of both CIN2+ and CIN3+ and significantly higher PPV for both CIN2+ and CIN3+, compared to women with medium protection, probably reflecting a higher background risk as well as lack of the screening effect in the women who did not participate in screening for a longer time.

\section{Response}

The response rates in all study groups were higher than the pooled response rates in intervention groups of the most recent meta-analysis on the subject. ${ }^{11}$ In our study, the response rates were $37.7 \%$ (95\% CI: 36.7-38.6), 34.0\% (95\% CI: 33.2-34.8), and $18.4 \%$ (95\% CI: 16.9-19.9) in the opt-out, opt-in and control groups, respectively, compared to the meta-analysis, where the response rates were $23.6 \%$ (95\% CI: 20.2-27.3), 14.0 \% (95\% CI: 8.0-21.4), 10.3\% (95\% CI: 6.2-15.2\%) in the opt-out, opt-in, opt-out control and opt-in control approach, respectively. ${ }^{11}$ Additionally, some previously published randomised studies reported similar response rates in the opt-out and control groups, e.g. $34.2 \%$ and $17.6 \%$, respectively, in the Netherlands ${ }^{19}$, and $39.0 \%$ in the opt-out group in a Swedish study. ${ }^{20}$ The opt-in responses recorded in our study were also in line with a recently published randomised Danish study (30.9\%) and a less powered Swedish study $(24.5 \%)$, which are, to the best of our knowledge, the only published randomised studies that showed a significantly higher response in the optin group than in the control group; however, the second study was without the opt-out group. ${ }^{16,17}$ Additionally, high overall response rates in opt-in groups were reached in some previous non-randomised studies, e.g. 39.1\% in the Swedish ${ }^{22}$ and $34.2 \%$ in the Danish study. ${ }^{23}$

A high response rate obtained in the opt-in group (close to one obtained in the opt-out group, but still significantly lower) is probably the most important finding of our study. Thus, the opt-in/ control group response ratio was significantly higher in our study $(1.8 ; 95 \%$ CI: $1.7-2.0)$ than the one recorded in the most recent meta-analysis (0.97; 95\% CI: 0.65-1.46). ${ }^{11}$ High overall response in the opt-in group is a consequence of high response to cytology, since in the opt-in group, more women attended the screening with a PG than performed the HPV self sampling. A relatively high response rate to cytology was also observed in some other opt-in ${ }^{15,16,23}$ and opt-out studies ${ }^{24}$; however, the HPV 
self-sampling response rate in those studies was always higher than the response rate to cytology. To our knowledge, there is only one recent study that obtained the higher response rate to cytology. ${ }^{17}$ In contrast, the opt-out/control group response ratio in our study was comparable to the pooled results of previous studies (our study: 2.0; 95\% CI: 1.9-2.2 vs meta-analyses: $2.4 ; 95 \%$ CI: $1.7-3.3) .{ }^{11}$

A high response in the opt-in group documented in our study could be explained by several factors. First, we provided women with a choice and encouraged them to either order a tester for HPV testing or make an appointment with a PG. They could have made an appointment by themselves, but in the case that they returned the questionnaire stating that they would prefer screening with a PG, we sent them a regular invitation letter and due to local health insurance rules, PGs must screen women with such an invitation not later than three months after the request for a screening appointment. Second, since women in the opt-in group had to devote more effort to undergo screening (they had to order a tester), we sent them two reminders to order the tester. Therefore, women in the opt-in study group could have received up to five invitation letters (the initial invitation letter, two reminders for ordering the tester, the letter with the tester package, and a reminder to return the sample), compared to women in the opt-out group, who according to the study protocol could have not received any reminders to order the tester. We used each contact as an opportunity to inform women why it was important to perform the screening and provided them with the option to communicate with us via a survey, telephone or email. Third, cytology and the HPV testing responders had different timelines for a response. ${ }^{25}$ One-year follow-up time in our study provided women with enough time to arrange their appointment and attend the screening with a PG. In comparison to our study, follow-up times in some of the other randomised studies were shorter (starting from 3 months) ${ }^{11}$, thus the full cytological response might not have yet been achieved. Fourth, mandatory recording of cytological results in the central screening registry enabled us to trace the clear majority of cytological results and to assess this type of response reliably.

\section{Histological outcomes}

CIN2+ was diagnosed in 3.6\% (86/23.956-95\% CI: $2.9 \%-4.5 \%$ o) of all the women enrolled in the intervention groups and in $10.1 \%$ o $(86 / 8.494-95 \% \mathrm{CI}$ : $8.2 \%-12.6 \%$ o) of all responders in the intervention groups. These results are in line with the recent meta-analysis where the corresponding probabilities were $2.9 \%$ o (95\% CI: $1.6-4.6 \%$ ) and 9.3\% (95\% CI: $6.8-12.1 \%$ o). ${ }^{11}$ We didn't record any significant difference in CIN2+ outcomes between the study groups; however, the probability for the CIN2+ diagnosis after the enrolment was higher in the optout group $(3.9 \%$ ) and the opt-in group $(3.4 \%$ ) than in the control group $(3.1 \%)$. This is in line with previous studies that suggested that CIN2+ outcomes among all the enrolled women were related to the response rate and were, due to a higher response, higher in opt-out self-sampling groups than opt-in and control groups. ${ }^{11}$ Our study might be underpowered for the detection of high-grade lesions, since it was scaled to detect difference in the response rates. However, it should be noted that we observed an insignificantly higher detection rate of CIN2+ among the women who performed cytology with PG in both intervention groups $(12.6 \%$ in opt-in and $14.2 \%$ in opt-out), compared to women with the HPV testing (8.4\%o and $10.3 \%$ ) (data not shown). If the difference is real, that could be explained either as a high sensitivity of cytological screening by PGs and/or that women with a higher risk for CIN2+ (potentially some of them already symptomatic) preferred screening by PGs over HPV testing on self-collected samples. It should be noted that $7 / 10$ women with cervical carcinoma who were enrolled in the intervention arm preferred cytological screening with PG; in four of them, the FIGO stage at diagnosis was IIB+. Only $3 / 10$ women with cervical carcinoma preferred selfsampling (FIGO stages IA1, IA2 and IIB). It should also be emphasised that no single case of CIN2+ was detected among 612 women who preferred screening with both HPV self-sampling and PG, which might be explained as a low risk of disease among the women with negative HPV test among the women who overuse health interventions compared to women who don't. ${ }^{26}$

\section{Results of HPV testing on self-collected samples}

HPV positivity rate among women who performed HPV self-sampling was 8.3\% (95\% CI: 7.5\%-9.0\%), which is significantly lower than in a recent metaanalysis (10.5\%, 95\% CI: $9.1 \%-12.9 \%) .{ }^{11}$ It is well known that HPV positivity rate is country- and age-specific. Lower HPV positivity rate recorded in our study could be related to a relatively high mean age of the HPV self-sampling responders, which was 50.3 years. In comparison to the results 
of a Slovenian study performed among 4.431 women aged 20-64 who regularly attended the national cervical cancer screening programme, we recorded a higher HPV positivity rate in all age groups except in the oldest women, aged 60-64 years. ${ }^{27}$ This can be explained either as a higher risk of HPV infection and cervical disease among non-attenders in the screening programme or as the detection of vaginal HPV infections in self-sampling samples that do not necessarily correlate with cervical HPV infections detected in practitioner-obtained cervical samples. A higher HPV positivity rate due to the detection of vaginal infections with low-risk HPV types might limit the cost-effectiveness of self-sampling due to a higher referral; however, it should be emphasised that one woman with a positive HPV test was diagnosed with VaIN3.

\section{Compliance with a follow-up examination after a positive HPV test}

The compliance with a follow-up examination in our study was $90.2 \%$, which is at the upper edge of the $95 \%$ confidence interval of the recent metaanalysis, where the compliance was $82.2 \%$ (95\% CI; 65.8-94.4). ${ }^{11}$ The achieved high compliance was most probably the result of prescheduled appointments, personal contacts via telephone with women who did not attend, and two prescheduled reminders. In our study the compliance was significantly related to the region of residence and the level of protection, so a different local context and the difference in the previous screening history might have contributed to the heterogeneity of results in various studies.

The concordance of positive results of HPV testing performed on self-collected samples and on practitioner-obtained cervical samples was $43.8 \%$ compared to $52.0 \%$ in a recent Norwegian study (considering only $\mathrm{HC} 2$ results) ${ }^{28}, 58.7 \%$ and $68.8 \%$ in Dutch studies ${ }^{29,30}$ and $73.3 \%$ in Swedish study. ${ }^{22}$ The concordance is also associated with the time difference between the two samplings, which mean value was 57 days in our study; during this time, some transitional HPV infections might have cleared. Concordance could be lower also due to the detection of vaginal HPV infections in self-collected samples.

\section{Positive predictive value of HPV testing on self-collected samples}

PPV of a test or testing strategy is important for justifying further diagnostic and therapeutic pro- cedures and can be used in risk-based management approach. If the risk is low for the underlying disease after a positive HPV test (PPV for CIN2+ $<2 \%$ and $\mathrm{CIN} 3+<3 \%$ ), the woman can be referred to regular screening. ${ }^{13,31}$ If the risk of the underlying disease is high (PPV for CIN2+ $>20 \%$ and CIN3+ $>10 \%$ ), the woman should be referred to colposcopy. Women with an intermediate risk should be triaged or followed up. In our study, the PPV of the HPV testing on self-collected samples was $12.0 \%$ for $\mathrm{CIN} 2+$ and $9.6 \%$ for $\mathrm{CIN} 3+$, which represents an intermediate risk with PPV for CIN3+, close to the cut-off for high risk. The women with a positive result of the second HPV test, performed on the practitioner-obtained cervical sample taken after mean of 57 days after a positive initial HPV testing had a much higher risk for the underlying disease, which justified immediate colposcopy (PPV was $23.3 \%$ for CIN2+ and $19.9 \%$ for CIN3+). However, there was a subgroup of women, with no/low protection due to a previous screening history, where the PPV of the HPV testing on self-collected samples was high enough (20.0\% for CIN2+ and $18.2 \%$ for CIN3+) to justify immediate colposcopy. This could imply that more than one management strategy would be appropriate for the women with an initially positive HPV testing on self-collected samples.

\section{Strengths and limitation of the study}

The key strengths of our study were a large sample size and the fact that the study was performed in the setting of an organised routine cervical cancer screening programme, which allows assessment of the efficacy as well as effectiveness of a possible upgrade to the Slovenian national screening programme with HPV self-sampling for non-attenders with efficient triage strategy. To the best of our knowledge, this is one of the first randomised studies among non-attenders of an organised screening programme with three study groups: the opt-in, the opt-out and the classic reminders approach, which achieved a relatively high response in the opt-in scenario compared to the opt-out scenario. The randomised study design with predetermined possible predictors for response and the risk of disease will allow us to identify the benefits and risks of both approaches: the opt-in and the opt-out, the best strategy to upgrade the programme, and the related costs. Because colposcopy was done per the protocol in women with a positive HPV results, PPV was computed for different subgroups of women. This will allow informed evidence-based decisions regarding the risk-stratified diagnos- 
tic and treatment follow-up of women who had a positive result of HPV testing on self-collected samples.

The main limitations of our study were that women with a positive result of the HPV testing had more intensive per protocol diagnostic followup (including colposcopy) than women with abnormal cytology, who were managed according to the national guidelines. This may account for the verification bias for histological outcomes when comparing the results of the HPV testing on selfcollected samples to cytology with PG or to previous studies which did not include an extensive colposcopy. However, the proportion of women with histology result in one year after their enrolment was similar and even higher in type $\mathrm{B}$ responders (4.0\% of responders) compared to $3.4 \%$ in type $\mathrm{A}$ responders, and the detection rates of CIN2+ and CIN3+ lesions between type A and type B responders did not differ significantly (data not shown). The main reason for more intensive per protocol diagnostic follow-up for women with positive result of the HPV test was to reliably assess the risk for the underlying high-grade disease among those women. Furthermore, an additional cervical sample taken during a follow-up examination will enable the comparison of different triage strategies for women with a positive HPV result. We initially included some women who appeared to be eligible at the time of their enrolment, but were later found to be non-eligible due to hysterectomy and recent cytology with a PG, but not yet registered in the ZORA registry. We, however, anticipated such situation, which will also certainly be present during future real-life HPV self-sampling of non-attenders. Because of this and the randomised nature of our study, we did not compute corrected response rates, and histological results and all analyses were made on the intention-to-screen basis (with all the enrolled women in the denominator).

\section{Conclusions}

In conclusion, an upgrade of the Slovenian cervical cancer screening programme ZORA with opt-out or opt-in HPV self-sampling could lead to a higher coverage of the target population. Also, stratified management of women with a positive result of the HPV testing on self-collected samples, according to their characteristics and background risk for the high-grade disease, should be strongly considered. Our study showed that high response rate could also be reached in opt-in settings if women were given a choice to attend the screening with the PG or order tester for HPV self-sampling and if access to screening is free and available without significant additional effort. Our results suggest that in real-life screening settings, the opt-in approach might be almost as effective in the overall high-grade disease detection among all the eligible women as the opt-out approach while being superior regarding cost-effectiveness. However, an indepth analysis should be done to identify the most cost-effective HPV self-sampling approach in the local setting. Although the difference in PPVs of the HPV testing for high-grade disease among specific subgroups of women in our study supports the concept of risk-based management of women ${ }^{31}$, further in-depth analysis is needed to clarify the role of potential PPV predictors after a positive result of HPV testing on self-collected samples.

\section{Acknowledgment}

The authors would like to thank Mojca Kuster from the NP ZORA coordination centre at the Institute of Oncology Ljubljana for her kind and professional communication with the enrolled women and staff at both colposcopy clinics and for the very much appreciated help with the study logistic. Special thanks goes to the staff of the colposcopy units at all three institutions: Tatjana Kodrič, Andrej Cokan, Sarah Dobnik, Jure Knez, Marica Miklavc, Marcela Živko, Aleksandra Muhič, Sonja Bebar, Sebastjan Merlo and Marta Janežič. We would also like to thank the staff from cytology and molecular laboratories at University Clinical Centre Maribor, General Hospital Celje and Institute of Oncology Ljubljana for their valuable work an contribution to the study: Nina Irgel, Lidija Salobir, Nevenka Štiglic Toš, Ana Katarina Seher, Branko Antolovič, Maja Fras, Barbara Jelen, Patricija Pernat, Saša Praznic, Živa Pohar Marinšek, Sandra Jezeršek, Simona Uhan Kastelic, Janja Zalar, Mojca Lešnjak, Simon Buček, Uršula Prosenc Zmrzljak, Marina Bučić, Slavica Vuzem and Barbara Verk. Special thanks also to a team of epidemiologists from Epidemiology and Cancer Registry unit at the Institute of Oncology Ljubljana for the initial support with setting up the project database and discussions regarding the study protocol. Last but not least, we also thank all the women who participated in the study.

Study was approved for public funding by the Slovenian Research Agency (ARRS) (project number L3-5512). It was financed by ARRS and Ministry of 
Health of Republic of Slovenia. Materials used in the study were obtained free of charges or with discount from manufacturers. ARRS, Ministry of Health and manufacturers didn 't have any role in the design of the study, study execution, analyses, interpretation of the data, or decision to submit results.

\section{References}

1. International Agency for Research on Cancer (2005). Cervix Cancer Screening. IARC handbooks of cancer prevention, Vol. 10. Lyon: IARCPress. [cited $2018 \mathrm{Apr}$ 15]. Available from: http://www.iarc.fr/en/publications/ pdfs-online/prev/handbook10/index.php

2. Vaccarella S, Lortet-Tieulent J, Plummer M, Franceschi S, Bray F. Worldwide trends in cervical cancer incidence: impact of screening against changes in disease risk factors. Eur J Cancer 2013; 49: 3262-73. doi: 10.1016/j. ejca.2013.04.024

3. Zadnik V, Primic Zakelj M, Lokar K, Jarm K, Ivanus U, Zagar T. Cancer burden in Slovenia with the time trends analysis. Radiol Oncol 2017; 51: 47-55. doi: 10.1515/raon-2017-0008

4. Spence AR, Goggin P, Franco EL. Process of care failures in invasive cervical cancer: systematic review and meta-analysis. Prev Med 2007; 45: 93-106. doi: 10.1016/j.ypmed.2007.06.007

5. Sigurdsson K, Hrafnkelsson J, Geirsson G, Gudmundsson J, Salvarsdóttir A. Screening as a prognostic factor in cervical cancer: analysis of survival and prognostic factors based on Icelandic population data, 1964-1988. Gynecol Oncol 1991; 43: 64-70.

6. Takac I, Ursic-Vrscaj M, Repse-Fokter A, Kodric T, Rakar S, Mozina A, et al. Clinicopathological characteristics of cervical cancer between 2003 and 2005, after the introduction of a national cancer screening program in Slovenia. Eur J Obstet Gynecol Reprod Biol 2008; 140: 82-9. doi: 10.1016/j. ejogrb.2008.02.019

7. Landy R, Pesola F, Castañón A, Sasieni P. Impact of cervical screening on cervical cancer mortality: estimation using stage-specific results from a nested case-control study. Br J Cancer 2016; 115: 1140-46. doi: 10.1038/ bjc. 2016.290

8. [National Cervical Cancer Screening Programme ZORA.] [Slovenian]. DP ZORA: Kazalniki. [online] https://zora.onko-i.si/ [Accessed 2018 Mar 29] Available at: https://zora.onko-i.si/publikacije/kazalniki/

9. Elfstrom KM, Arnheim-Dahlstrom L, von Karsa L, Dillner J. Cervical cancer screening in Europe: quality assurance and organisation of programmes. Eur J Cancer 2015; 51: 950-68. doi: 10.1016/j.ejca.2015.03.008

10. Ivanuš U, Primic Žakelj M. [HPV self-sampling among non-responders to cervical cancer screening programmes: systematic review]. [Slovenian]. Onkologija 2012; 2: 78-86. Available also online (in Slovenian language only): https://www.onko-i.si/fileadmin/onko/datoteke/dokumenti/ Onkologija_Letnik XVI st_2/7 Onkologija_2 2012 samoodvzem.pdf

11. Verdoodt F, Jentschke M, Hillemanns P, Racey CS, Snijders PJ, Arbyn M Reaching women who do not participate in the regular cervical cancer screening programme by offering self-sampling kits: a systematic review and meta-analysis of randomised trials. Eur J Cancer 2015; 51: 2375-85. doi: 10.1016/j.ejca.2015.07.006

12. Von Karsa L, Arbyn A, De Vuyst H, Dillner J, Dillner L, Franceschi S, et al. (2015). Executive summary. In: European guidelines for quality assurance in cervical cancer screening. Second edition, Supplements. Anttila A, Arbyn A De Vuyst H, Dillner J, Dillner L, Franceschi S, et al, editors. Luxembourg: Office for Official Publications of the European Union. p. XIII-XXIV. [cited $2018 \mathrm{Apr}$ 10]. Available from: https://publications.europa.eu/en/publication-detail/-/ publication/a41a4c40-0626-4556-af5b-2619dd1d5ddc/language-en

13. Arbyn M, Verdoodt F, Snijders PJ, Verhoef VM, Suonio E, Dillner L, et al. Accuracy of human papillomavirus testing on self-collected versus cliniciancollected samples: a meta-analysis. Lancet Oncol 2014; 15: 172-83. doi 10.1016/S1470-2045(13)70570-9

14. Giorgi Rossi P, Marsili LM, Camilloni L, lossa A, Lattanzi A, Sani C, et al; SelfSampling Study Working Group. The effect of self-sampled HPV testing on participation to cervical cancer screening in Italy: a randomised controlled trial (ISRCTN96071600). Br J Cancer 2011; 104: 248-54. doi: 10.1038/ sj.bjc. 6606040
15. Giorgi Rossi P, Fortunato C, Barbarino P, Boveri S, Caroli S, Del Mistro A, et al. HPV Self-sampling Italian Working Group. Self-sampling to increase participation in cervical cancer screening: an RCT comparing home mailing, distribution in pharmacies, and recall letter. Br J Cancer 2015; 112: 667-75. doi: 10.1038/bjc.2015.11

16. Broberg G, Gyrd-Hansen D, Miao Jonasson J, Ryd ML, Holtenman M, Milsom I, et al. Increasing participation in cervical cancer screening: offering a HPV self-test to long-term non-attendees as part of RACOMIP, a Swedish randomized controlled trial. Int J Cancer 2014; 134: 2223-30. doi: https://doi. org/10.1002/ijc.28545

17. Tranberg $M$, Bech BH, Blaakær J, Jensen JS, Svanholm H, Andersen B. Preventing cervical cancer using HPV self-sampling: direct mailing of testkits increases screening participation more than timely opt-in procedures - a randomized controlled trial. BMC Cancer 2018; 18: 273. doi: 10.1186/ s12885-018-4165-4

18. $\mathrm{R}$ Core Team. R: A language and environment for statistical computing Vienna: R Foundation for Statistical Computing. 2012. ISBN 3-900051-07-0, URL. Available from: http://www.R-project.org/

19. Ursic-Vrscaj M, Rakar S, Možina A, Kobal B, Takač I, Deisinger I, et al. [Guidelines for management of women with cervical precancerous lesions]. [Slovenian] In: Ursic-Vrscaj M, editor. Ljubljana: Institute of Oncology Ljubljana; 2011. [cited 2018 Mar 15]. Available at (in Slovene language only): https://zora.onko-i.si/fileadmin/user_upload/dokumenti/strokovna_priporocila/2011_Smernice_web.pdf

20. Bais AG, van Kemenade FJ, Berkhof J, Verheijen RH, Snijders PJ, Voorhorst F, et al. Human papillomavirus testing on self-sampled cervicovaginal brushes: an effective alternative to protect nonresponders in cervical screening programs. Int J Cancer 2007; 120: 1505-10. doi: 10.1002/ijc.22484

21. Wikström I, Lindell M, Sanner K, Wilander E. Self-sampling and HPV testing or ordinary Pap-smear in women not regularly attending screening: a randomised study. Br J Cancer 2011; 105: 337-9. doi: 10.1038/bjc.2011.236

22. Sanner K, Wikström I, Strand A, Lindell M, Wilander E. Self-sampling of the vaginal fluid at home combined with high-risk HPV testing. Br J Cancer 2009; 101: 871-4. doi: 10.1038/sj.bjc.6605194

23. Lam JU, Rebolj M, Møller Ejegod D, Pedersen H, Rygaard C, Lynge E, et al. Human papillomavirus self-sampling for screening nonattenders: opt-in pilot implementation with electronic communication platforms. Int $J$ Cancer 2017; 140: 2212-19. doi: 10.1002/ijc.30647

24. Cadman L, Wilkes S, Mansour D, Austin J, Ashdown-Barr L, Edwards R, et al. A randomized controlled trial in non-responders from Newcastle upon Tyne invited to return a self-sample for Human Papillomavirus testing versus repeat invitation for cervical screening. J Med Screen 2015; 22: 28-37. doi: $10.1177 / 0969141314558785$

25. Kitchener H, Gittins M, Cruickshank M, Moseley C, Fletcher S, Albrow R, et al. A cluster randomized trial of strategies to increase uptake amongst young women invited for their first cervical screen: The STRATEGIC trial. $J$ Med Screen 2017. doi: 10.1177/0969141317696518 [Epub ahead of print]

26. Dugué PA, Lynge E, Rebolj M. Mortality of non-participants in cervical screening: Register-based cohort study. Int J Cancer 2014; 134: 2674-82. doi: $10.1002 /$ ijc. 28586

27. Učakar V, Poljak M, Klavs I. Pre-vaccination prevalence and distribution of high-risk human papillomavirus (HPV) types in Slovenian women: a cervical cancer screening based study. Vaccine 2012; 30: 116-20. doi: 10.1016/j. vaccine.2011.10.066

28. Enerly E, Bonde J, Schee K, Pedersen H, Lönnberg S, Nygård M. SelfSampling for human papillomavirus testing among non-attenders increases attendance to the Norwegian Cervical Cancer Screening Programme. PLoS One 2016; 11: e0151978. doi: 10.1371/journal.pone.0151978

29. Gök M, van Kemenade FJ, Heideman DA, Berkhof J, Rozendaal L, Spruyt JW, et al. Experience with high-risk human papillomavirus testing on vaginal brush-based self-samples of non-attendees of the cervical screening program. Int J Cancer 2012; 130: 1128-35. doi: 10.1002/ijc.26128

30. Gök M, Heideman DA, van Kemenade FJ, Berkhof J, Rozendaal L, Spruyt JW, et al. HPV testing on self collected cervicovaginal lavage specimens as screening method for women who do not attend cervical screening: cohort study. BMJ 2010; 340: c1040. doi: 10.1136/bmj.c1040. Erratum in: BMJ 2016; 353: i2823.

31. Castle PE, Sideri M, Jeronimo J, Solomon D, Schiffman M. Risk assessment to guide the prevention of cervical cancer. Am J Obstet Gynecol 2007; 197: 356.e1-6. doi: 10.1016/j.ajog.2007.07.049 